Recepción: 20 / 12 / 2017

Aceptación: 15 / 02 / 2018

Publicación: 21 / 04 / 2018

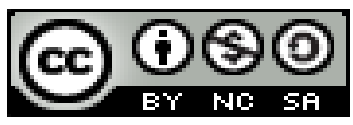

Ciencias Químicas

Artículo de Revisión

\title{
Presencia de Microorganismos patógenos en las aguas del Estero Salado año 2013
}

\section{Presence of pathogenic microorganisms in the waters of Estero Salado year 2013}

\section{Presença de microrganismos patogênicos nas águas de Estero Salado, ano de 2013}

\author{
Walter E. Mariscal-Santi ${ }^{\text {I }}$ \\ walter.mariscals@ug.edu.ec \\ Frella S. Garcia-Larreta II \\ frella.garcia@hotmail.com \\ Raisa S. Mariscal-Garcia III \\ raisamariscal@hotmail.com \\ Cinthia A. Paredes-Jara IV \\ cinthia_amabel@hotmail.com
}

Henry X. Ponce-Solórzano V henry.ponces@ug.edu.ec

\author{
Troski A. Montiel-Rivera VI \\ troskymontielrivera@gmail.com
}

Eduardo F. De La Torre-Quinonez VII

eduardofrancisco.delatorre@gmail.com

Correspondencia: walter.mariscals@ug.edu.ec

\footnotetext{
I Docente, Universidad de Guayaquil, Guayaquil, Ecuador.

II Docente, Universidad de Guayaquil, Guayaquil, Ecuador.

${ }^{\mathrm{III}}$ Docente, Universidad de Guayaquil, Guayaquil, Ecuador.

${ }^{\text {IV }}$ Docente, Universidad de Guayaquil, Guayaquil, Ecuador.

${ }^{v}$ Docente, Universidad de Guayaquil, Guayaquil, Ecuador.

${ }^{\text {VI } D o c e n t e, ~ U n i v e r s i d a d ~ d e ~ G u a y a q u i l, ~ G u a y a q u i l, ~ E c u a d o r . ~}$

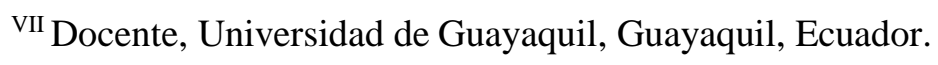




\section{Resumen}

La contaminación ambiental es un problema sanitario que empeora cada día en nuestra sociedad debido a la carencia de labores de remediación ambiental por parte de los gobiernos a predominio de los países subdesarrollados. La presencia de microorganismos patógenos en las aguas, es un indicador fiel de contaminación, por la presencia de excretas de origen animal o humano. En el Estero Salado Norte de la ciudad de Guayaquil se decidió realizar la determinación de microorganismos patógenos en vista de constituir este parte del pulmón vegetal y acuífero de la Ciudad de Guayaquil. Métodos: se tomó muestras de tres diferentes puentes analizando en laboratorios la presencia de microorganismos patógenos en el agua, por un periodo de 3 meses. Resultados: Se obtuvo altos niveles de contaminación de las aguas por presencia de microorganismos coliformes relacionados con la inadecuada disposición de excretas animales y humanas en el área. Discusión: con los resultados obtenidos, se aprecia la imperiosa necesidad de iniciar planes sanitarios que permitan el saneamiento de las aguas, en vista de su cercanía y uso por parte de las poblaciones circunvecinas.

Palabras clave: Aguas contaminadas, microorganismos coliformes, guayaquil, estero salado. 


\section{Abstract}

Environmental pollution is a health problem that worsens every day in our society due to the lack of environmental remediation work by governments in the predominance of underdeveloped countries. The presence of pathogenic microorganisms in the water is a faithful indicator of contamination, due to the presence of excreta of animal or human origin. In the North Estero Salado of the city of Guayaquil it was decided to carry out the determination of pathogenic microorganisms in view of constituting this part of the plant lung and aquifer of the City of Guayaquil. Methods: Samples were taken from three different bridges, analyzing in laboratories the presence of pathogenic microorganisms in the water, for a period of 3 months. Results: High levels of water contamination were obtained due to the presence of coliform microorganisms related to the inadequate disposal of animal and human excreta in the area. Discussion: with the results obtained, we can see the imperative need to start sanitary plans that allow the sanitation of the waters, in view of their proximity and use by the surrounding populations.

Keywords: Contaminated waters, coliform microorganisms, guayaquil, estero salado 


\section{Introducción.}

El agua es el compuesto químico más abundante en la superficie terrestre y a la vez el más importante para la vida. Está presente en grandes cantidades en la naturaleza, ya sea en estado sólido, líquido o gaseoso, el agua se encuentra formando parte vital de los seres vivos, en ella viven y se multiplican los organismo más simples y los más complejos contribuyendo de esta manera a la formación de los distintos líquidos necesarios para sus procesos biológicos. (ACOSTA, ZAPATA, \& FAGUA, 2009). El agua se encuentra formando parte de los océanos, ríos, lagos, glaciares y esteros, a la vez forma parte de las rocas dentro del subsuelo o también en la atmosfera en forma de nubes o niebla; todo aquello representa las siete décimas partes de la superficie de la tierra, además constituye el $60 \%$ del cuerpo humano y hasta el $95 \%$ del peso de los vegetales. (Glynn \& Heinke, 1999). El término contaminación proviene del latín contaminare, cuyo significado es mezclar o también infectar, ensuciar, manchar. La contaminación ambiental en ríos y mares, es un problema que se deriva de las malas técnicas de disposición de aguas negras, del indiscriminado vertido de material de desecho industrial y de la ausencia de programas apropiados de remediación ambiental. Más del $60 \%$ de la población mundial, reside dentro de la zona costera, este porcentaje, se ha proyectado incrementarse hasta en un $80 \%$ (Harrison, 1999); de tal manera que esta tendencia ha conducido a problemas como incremento de desechos domésticos, municipales e industriales; ejerciendo mayor presión en los sistemas costeros, conllevando a problemas y forzando aún más la capacidad límite de las plantas de tratamiento de aguas servidas.

La contaminación por microorganismos patógenos puede ser de diferentes tipos bacteriana,

viral, por protozoos y otros organismos que transmiten enfermedades como el cólera, tifus, gastroenteritis diversas, hepatitis, etc. En los países en vías de desarrollo las enfermedades producidas por estos patógenos son uno de los motivos más importantes de muerte prematura, sobre 
todo de niños. Normalmente estos microbios llegan al agua en las heces y otros restos orgánicos que producen las personas infectadas. Por esto, un buen índice para medir la salubridad de las aguas, en lo que se refiere a estos microorganismos, es el número de bacterias coliformes presentes en el agua. La Organización Mundial de la Salud (OMS) recomienda que en el agua para beber haya 0 colonias de coliformes por $100 \mathrm{ml}$ de agua. Químicamente, los coliformes, son bacilos gramnegativo no esporulados, que puede desarrollarse en presencia de sales biliares u otros agentes tenso activos con similares propiedades de inhibición de crecimiento, no tienen citocromo oxidasa y fermentan la lactosa con producción de ácido, gas y aldehído a temperaturas de 44 o $44,5{ }^{\circ} \mathrm{C}$, en un periodo de 24 a 48 horas. También se les designa como Coliformes termo-resistentes o termo tolerantes. Las bacterias del grupo coliforme se encuentran en el intestino, en las heces humanas y en las de animales de sangre caliente. Los coliformes termotolerantes comprenden a los géneros de Escherichia y en menor grado Klebsiella, Enterobacter, Streptococcus, y Citrobacter. Los estreptococos fecales están constituidos por especie del genero Streptococcus como S. faecalis, S. faecium, S. avium, S. bovis, S. equim y S. gallinarum. (González Delgado, Orozco Barrenetxea, Pérez Serran, Alfayate Blanco, \& Rodriguez Vidal, 2005).

En vertidos domésticos de aguas residuales de alcantarillado, fosas sépticas, corrientes urbanas, granjas de animales y parques, goteos de aguas de aves y aplicaciones a la tierra de residuos de animales, la presencia de Coliformes en aguas superficiales, indica contaminación proveniente de residuos humanos, animales o erosión del suelo separadamente, o de una combinación de las tres fuentes.

Nuestro país no escapa de esta situación, es por ésta razón que se decide evaluar la presencia de microorganismos patógenos que tantos daños le ocasionan a la población circunvecina al Estero Salado del Sector norte de la Ciudad de Guayaquil. 
Este trabajo de investigación fue realizado con un enfoque descriptivo, explicativo, metodológico, cuantitativo y transversal; que constituya una propuesta a considerar en las diferentes acciones de remediación ambiental de este importante tramo del Estero Salado por parte de las entidades gubernamentales, municipales y ambientales, llegando a uno de los componentes más importantes de esta tarea como es la educación ambiental y social, por medio del cual se debe trabajar en la sensibilización y concienciación de la población para promover la participación y el empoderamiento de la comunidad vecina del estero.

\section{Materiales y métodos.}

La población a la que tiene alcance la investigación está constituida por las aguas del Estero Salado, sector norte de la ciudad de Guayaquil cuya ubicación donde se realiza el trabajo es desde el Primer Puente de Miraflores (Este:17 M 720174;Norte: 17M 9760938) continuando al Segundo Puente de Albán Borja (Este: 17 M 620534;Norte: 17 M 9760550), hasta el Tercer Puente de la Av. Víctor Emilio Estrada (Este: 17M 621961;Norte:9759265). En la zona superior de este tramo (Puente Miraflores), la sección del flujo es relativamente estrecha y se seca durante las horas de marea baja, en cambio en la parte inferior (Puente Av. V. E. Estrada), tiene un ancho aproximado de 60m y una profundidad promedio de 4m, (Lahmeyer-Cimentaciones,2000). Por consiguiente, la investigación que se realizó, permitió obtener la información básica de los parámetros físicoquímicos y microbiológicos de las aguas, para determinar una apropiada evaluación sobre el nivel de contaminación de este sistema estuarino.

La zona del norte del Estero Salado de la ciudad de Guayaquil para objeto del muestreo fue dividido en 9 puntos referenciados con GPS (Global Positionning System), modelo Garmn Etrex, para determinar la localización exacta (coordenadas) del área de investigación y en cada estación se medirán in situ y en el laboratorio (ex situ), los parámetros de la calidad del agua. 
La Medición es directa e indirecta, y permite determinar características microbiológicas del agua del estero saldo y sus resultados se logran gracias a una buena recolección de la muestra. Se recolectó muestras agua para los análisis microbiológicos en el laboratorio para determinar Coliformes totales, acompañados de Coliformes fecales y enterococos fecales expresados en Nmp/100ml. Se utilizó el método para analizar Coliformes fecales; Número Más Probable (MPN) de acuerdo al API-5.8-0401-00M27 y API-5.8-04-01-00M22 (Standard Methods 21th 9221 ABCE.

\section{Resultados.}

En la determinación de presencia en el agua de Coliformes por el método del Número Más Probable (Nmp) se determinó, en el mes de Octubre, que el puente de Miraflores presentó un promedio de 2.4 x 106, en el puente de Albán Borja 1.6 x 106 y en el puente de Urdesa 4.5 x 103 Nmp/100mL. de Coliformes totales.

En lo referente a Coliformes fecales, en el puente de Miraflores se obtuvo un promedio de 1.4 x 106, en el puente de Albán Borja, 2.5 x 106 y en el puente de Urdesa 2.1 x 106 Nmp/100mL y de Enterococos fecales, en el puente Miraflores registró un promedio de 1.8 x 106, puente Albán Borja 2.6 x 106 y en el puente de Urdesa 2.1 x 103 Nmp/100mL.

En el mes de Agosto, que el puente de Miraflores presentó un promedio de 2.2 x 106, en el puente de Albán Borja 1.7 x 106 y en el puente de Urdesa 4.9 x 103 Nmp/100Ml. de Coliformes totales. En lo referente a Coliformes fecales, en el puente de Miraflores se obtuvo un promedio de 1.1 x 106, en el puente de Albán Borja, 2.2 x 106 y en el puente de Urdesa 2.3 x 106 Nmp/100mL y de Enterococos fecales, en el puente Miraflores registró un promedio de 1.6 x 106, puente Albán Borja 2.4 x 106 y en el puente de Urdesa 2.5 x 103 Nmp/100mL. 
En el mes de Diciembre, que el puente de Miraflores presentó un promedio de 2.35 x 106 , en el puente de Albán Borja 1.8 x 106 y en el puente de Urdesa 4.2 x 103 Nmp/100Ml. de Coliformes totales. En lo referente a Coliformes fecales, en el puente de Miraflores se obtuvo un promedio de 1.6 x 106, en el puente de Albán Borja, 2.3 x 106 y en el puente de Urdesa 2.6 x 106 Nmp/100mL y de Enterococos fecales, en el puente Miraflores registró un promedio de 1.9 x 106, puente Albán Borja 2.5 x 106 y en el puente de Urdesa 2.4 x 103 Nmp/100mL. Estos valores están muy por encima de los estándares establecidos por las TULSMA.

\section{Discusión.}

Para el análisis de las aguas se utilizaron los estándares establecidos por las TULSMA e ICA para tener una guía de resultados permitidos en un cuerpo de agua clasificado. En el caso del estero salado sector norte no cualifica por sus condiciones de deterioro a causa de la contaminación existente. También hay que resaltar que las muestras y análisis se tomaron entre los meses de julio, agosto y septiembre, los cuales fueron no lluviosos y por la naturaleza de este sistema en flujo continuo, el estero no estuvo expuesto a limpieza y renovación de sus aguas constantemente.

La presencia de microorganismos coliformes es un indicador biológico de la descarga de materia orgánica de origen doméstico. Su presencia es evidencia de contaminación fecal, la mayoría de estos microorganismos son anaeróbicos y facultativos, pero otros dependen del oxígeno disuelto para realizar procesos de metabolización. Aunque no es posible distinguir entre Coliformes de origen humano o animal, existen ensayos para diferenciar entre Coliformes totales, que incluyen los de animales y suelo, y Coliformes fecales, y Enterococos fecales, que incluyen únicamente los humanos. 
Con relación a los parámetros microbiológicos, la cantidad de coliformes totales, Coliformes fecales y enterococos fecales, encontrados es preocupante para la calidad de vida de todos los seres humanos que tienen contacto con el área y directamente con el agua.

Comúnmente en esta población, se observa personas bañándose, y pescando en esta agua, lo que representa un problema sanitario de necesidad inmediata de resolución mediante la aplicación de programas sanitarios que garanticen la calidad del agua consumida en aras de evitar enfermedades a predominio del aparato gastrointestinal y de piel.

\section{Bibliografía.}

Acosta A. Zapata A.G., Fagua. Técnicas de campo en Ambientes tropicales. Manual para el Monitoreo en ecosistemas acuáticos. Bogotá Pontificia Universidad Javeriana.

APHA. 1998, Standard Methods for the Examination of Water and Wastewater.

Atlas, Ronald M.R. Bartha: Ecología Microbiana y Microbiología Ambiental. Trad R. Guerrero 4 ed Madrid. Prentice Hall.

Ayarza, W., Coello, S., Chalén, N., Garcés, P., García, L., García, M.L., Omarza, F., Pérez, E., Pesantes, F., Solórzano, L. 1993 «Estudios Geobioquímicos de la Sección Urbana del Estero Salado.» Revista de Ciencias del Mar y Limnología. Guayaquil-Ecuador.

CAAM 1996, Desarrollo y Problemática Ambiental del área del Golfo de Guayaquil-Ecuador.

Carvajal, R., M. Jiménez, y P. Iturralde, 2006. Zonificación de la Reserva de Producción de Fauna Manglares El Salado. Editado por Fundación Natura. Guayaquil-Ecuador.

Chalén, N. 1986. Mercurio en el estuario interior del Golfo de Guayaquil incluyendo el Estero Salado, Rio Guayas y los cursos inferiores de los Ríos Daule y Babahoyo, Guayaquil Instituto Nacional de Pesca-Ecuador.

Constitución de la República del Ecuador. Montecristi - Manabí, 25 de julio de 2008.

DePauw, N. 2007. Biological monitorig and assessment of surface water quality. Curso de monitoreo de la calidad de aguas. Escuela Superior Politécnica del Litoral, Guayaquil-Ecuador.

Downes, B. Fairweather, P. Kenough, M. and Mapstone. B. 2002. Monitoring ecological impacts, concepts and practice in flowing waters Cambridge University Press- Cambridge-Inglaterra.

Eginseco Cia. 2001. Diagnóstico y Plan Ambiental del Terminal Fuel Oil de Petrocomercial en la ciudad de Guayaquil-Ecuador. 
Empresa Municipal de Alcantarillado de Guayaquil (EMAG), 1980. Plan de Recuperación del Estero Salado primera parte. Guayaquil -Ecuador.

Enkerlin. 1997. Ciencia ambiente y desarrollo sostenible. México (MX): Thonsom Editores.

EPA. 2001. Nutrient Criteria Technical Guidance Manual. Estuarine and Coastal Marine Waters. United States Environmental Protection Agency Office of Water. Disponible -USA.

Estrella, T. 2000. Uso del Recurso Agua y Manglares en el Estero de Puerto Hondo. provincia del Guayas, Ecuador. tesis IV Maestria en conservación y gestión del medio natural: Integración de sistemas naturales y humanos. Guayaquil-Ecuador.

FAO 1981. Manual of Methods in aquatic environment research. Part 8, Ecological assessment of pollution effects (Guidelines for the FAO (GFCM)/UNEP Joint Coordinated Project on Pollution in the Mediterranean) Rome-Italy.

Fernández, C. 2005. Evaluación de Impacto Ambiental del Proyecto Centro Recreativo Balneario Puerto Hondo Parroquia tarqui, Guayaquil Fundación Guayaquil Siglo XXI-Ecuador.

Glynn. 1999. Ingeniería ambiental. México (MX): Editorial Prentice Hall.

Harrison. 1999. Entendiendo nuestro ambiente: Una introducción a la química ambiental y la contaminación. España (ES): Editorial Acribia.

Hartman, 1996, Atlas of The Ssdentariate Polychaetous Annelids From California, Allan Hancock Foundation. Los Ángeles, California, EEUU.

Hidroestudios, 2001: Plan Integral de Recuperación el Estero Salado Fase 1, Guayaquil, Estudios de impacto Ambiental de los desvíos temporales de los tramos A y B del Estero Salado. GuayaquilEcuador.

Holland, A.F., y otros, 2004. Linkages between Tidal Creek Ecosystems and the Landscape and Demographic Attributes of their Watersheds. Journal Experimental Marine Biology and Ecology. USA.

Holland, A.F.; Sanger, D.M.; Gawle, C. P.; Lerberg, S.B.; Santiago, M.S.; Riekerk, G.H.M.; Zimmerman, L.E.; Scott, G.E. 2004. «Journal Experimental Marine Biology and Ecology. » Linkages between Tidal Creek Ecosystems and the Landscape and Demographic Attributes of their Watersheds. USA.

Instituto Nacional de Estadística y Censos (INEC). Censo de población y vivienda, 2010 .Ecuador.

Lahmeyer-Cimentaciones, 2000. «Estudios de Prefactibilidad, Factibilidad y Selección de la Mejor Alternativa del PIRES.» Parte II, Informe Final, Preparado para la Muy Ilustre Municipalidad de Guayaquil. Guayaquil - Ecuador.

LEY DE AGUAS.- República del Ecuador.- 20 de Mayo de 2004.

McGraw-Hill. 1990. Diccionario de Química. Tomo II. McGraw-Hill/Interamericana de México.

Manahan. 2007. Introducción a La química ambiental. México (MX): Editorial Reverte UNAM. 
Medina, J. Monserrate, L. P. Calle. Evaluación de las condiciones químicas, físicas y biológicas del Estero Salado en el sector dentro de la ciudad de Guayaquil. Escuela Superior politécnica del Litoral. Guayaquil-Ecuador.

Ministerio del Ambiente. 2002. Texto Unificado de Legislación Ambiental Secundaria. edición Especial. Corporación de estudios y Publicaciones. Quito-Ecuador.

M.I. Municipalidad de Guayaquil, 2007. «Plan de Manejo Reserva de Producción Faunística Manglares el Salado.» Guayaquil-Ecuador.

Montaño, M. 2007. Estudio de la calidad del ambiente Estero Santa Ana-Salado frente a Trinipuerto. Auditoría Ambiental de cumplimiento. Transpuerto S.A. Dirección de Medio Ambiente del Municipio de Guayaquil-Ecuador.

Mora, E., Reinoso, B. 1981. «Investigaciones Preliminares sobre el estado actual de las poblaciones de ostiones en tres zonas del Estuario Interior del Golfo de Guayaquil.» Revista de Ciencias del Mar y Limnolo- Guayaquil-Ecuador

Murgel M. 1984. Linmología sanitaria estudio de la polución de aguas continentales. Estados Unidos (USA): Organización de Estados Americanos.

Murray, S. D. A. Simpong and J. Santoro 1973. Circulation and Salinity distribution in the Rio Guayas. Estuary Ecuador. Coastal Estudies, L.S.U. USA.

Orozco et al. 2005. Contaminación ambiental - una visión desde la química. España (ES): Thompson Editores.

Pesantes, F.E. Pérez 1993. Estudio comparativo de las condiciones Físicas y Químicas de las aguas costeras ecuatorianas. 1982-1983. Revista Ciencias del Mar. Linmología.- Guayaquil-Ecuador.

Perdomo C, Casanova O, \& Ciganada V. 2001. Contaminación de aguas subterráneas con nitratos y coniformes en el litoral sudoeste del Uruguay. Agrociencia vol. $\mathrm{N}^{\circ} 1$.

Primer Simposio de Biodiversidad Marina y Costera de Latinoamérica y el Caribe y Segundo Simposio Nacional de Biodiversidad Marina y Costera. 2010. Diferencias en distribución y abundancia de macro invertebrados bentónicos en sectores del Estero Salado con diferente grado de influencia antropogénica. Manta - Ecuador.

Rivera, J., 2007. Reserva de Producción de Fauna Manglares el Salado. Quito 1- Ecuador.

Ridgely and Greenfield (2001) the Birds of Ecuador. Especies de rango restringido, listadas para la región de los Andes del sur.

Rodier, J. 1981. Análisis de las Aguas. Aguas naturales, aguas residuales y agua de mar. BarcelonaEspaña.: Omega.

Ruiz et al. 2006. Determinación de la demanda química de oxígeno y su importancia en la ingeniería ambiental. En 2o foro académico nacional de Ingenierías y Arquitectura- México.

Sawyer, C. McCarthy, P. and Parkin, G. 2000. Química para la Ingeniería Ambiental. Tracd McGarw-Hill. 4 ed. Bogotá. Co. Interamericana. 
Soanez. 1999. Ingeniería del Medio Ambiente - Aplicada al medio ambiente natural continental. España (ES): Ediciones Mundi-Prensa.

Solórzano, L., Viteri, G., 1993. «Investigación Química del Estero Salado.» Revista de Ciencias del Mar y Limnología III.- Guayaquil-Ecuador.

Spiro \& Stigliani. 2004. Química medioambiental. España (ES): Editorial Pearson Educación S.A.

Tyler M. 2004. Ciencia Ambiental -Preservemos la tierra. México (MX): Editorial Thomsom.

Universidad de Guayaquil. 2008. Evaluación del nivel de contaminación del manglar y aguas del Estero Salado circundante a la Estación de Transferencia Tres Bocas de Petrocomercial Sur, con miras a establecer un plan de manejo Ambiental y de mitigación, Dirección de Investigaciones y Proyectos Académicos (DIPA) Facultad de Ingeniería Química, Departamento de Petróleo y Petroquímica..- Guayaquil-Ecuador.

Valencia, Manuel, 2001. Contaminantes del Estero Salado. Técnico, Guayaquil- Ecuador.

Vives De Andréis, José Benito, 2007. Manual de Técnicas Analíticas para la determinación de parámetros fisicoquímicos y contaminantes marinos (aguas, sedimentos y organismos). Programa Calidad Ambiental Marina - CAM. Ecuador.

Wiessman \& Hammer. 2005. Water supply and pollution control. Estados Unidos (USA): Pearson Prentice Hall Edition.

Wong and Wong, 2007. Estudio de Impacto Ambiental fase de operación, Terminal Portuario Internacional Puerto Hondo. Guayaquil-Ecuador.

Zambrano López, Nelson. 2007. Estudio de impacto ambiental del parque. recopilado por M. I. Municipalidad de Guayaquil. Ecuador.

Zambrano, N. 2007. Programa de Manejo de Recursos Costeros. Plan de Manejo del Bosque Protector Estero Salado. Guayaquil Ecuador. 